\title{
Upregulation of thioredoxin and its reductase attenuates arsenic trioxide-induced growth suppression in human pulmonary artery smooth muscle cells by reducing oxidative stress
}

\author{
WOO HYUN PARK \\ Department of Physiology, Research Institute for Endocrine Sciences, Medical School,
Chonbuk National University, Jeonju, Jeollabuk 54907, Republic of Korea \\ Received January 9, 2019; Accepted October 18, 2019
}

DOI: $10.3892 /$ or.2019.7414

\begin{abstract}
The thioredoxin (Trx) system is an important enzymatic complex involved in cellular redox homeostasis. Arsenic trioxide (ATO; $\mathrm{As}_{2} \mathrm{O}_{3}$ ) is known to trigger cell death in vascular smooth muscle cells (VSMCs) via oxidative stress. In the present study, the effects of changes in thioredoxin 1 (Trx1) and Trx reductase1 (TrxR1) on cell growth, death, reactive oxygen species (ROS), and glutathione (GSH) levels were evaluated in ATO-treated human pulmonary artery smooth muscle cells (HPASMCs). ATO inhibited growth and induced cell death in the HPASMCs at $24 \mathrm{~h}$. Overexpression of Trx 1 and TrxR1 using adenoviruses attenuated cell growth inhibition caused by ATO and partially prevented cell death. ATO increased ROS levels including the mitochondrial superoxide anion $\left(\mathrm{O}_{2}{ }^{-}\right)$at $5 \mathrm{~min}$. Administration of adTrx1 or adTrxR1 reduced the increased mitochondrial $\mathrm{O}_{2}{ }^{-*}$ level in these cells. HPASMCs treated with Trx1 or TrxR1 siRNA showed increases in ROS levels with or without treatment of ATO at $5 \mathrm{~min}$. Although ATO transiently increased GSH levels at 5 min, Trx 1 and TrxR1 siRNAs reduced the increased GSH
\end{abstract}

Correspondence to: Professor Woo Hyun Park, Department of Physiology, Research Institute for Endocrine Sciences, Medical School, Chonbuk National University, 20 Geonji-ro, Deokjin, Jeonju, Jeollabuk 54907, Republic of Korea

E-mail: parkwh71@jbnu.ac.kr

Abbreviations: VSMCs, vascular smooth muscle cells; HPASMCs, human pulmonary artery smooth muscle cells; ATO, arsenic trioxide $\left(\mathrm{As}_{2} \mathrm{O}_{3}\right)$; ROS, reactive oxygen species; Trx, thioredoxin; TrxR, Trx reductase; SOD, superoxide dismutase; GSH, glutathione; NADPH, nicotinamide adenine dinucleotide phosphate; PX-12, 1-methylpropyl 2-imidazolyl disulfide; MTT, 3-(4,5-dimethylthiazol-2-yl)-2,5-diphenyltetrazolium bromide; FBS, fetal bovine serum; FITC, fluorescein isothiocyanate; $\mathrm{H}_{2}$ DCFDA, 2',7'-dichlorodihydrofluorescein diacetate; DHE, dihydroethidium; CMFDA, 5-chloromethylfluorescein diacetate

Key words: vascular smooth muscle cells, arsenic trioxide, cell death, thioredoxin, reactive oxygen species, glutathione levels in these cells. In addition, PX-12 (a Trx1 inhibitor) and auranofin (a TrxR1 inhibitor) diminished the cellular metabolism in HPASMCs at $4 \mathrm{~h}$, accompanied by an increase in ROS level and a decrease in GSH level. In conclusion, upregulation of Trx1 and TrxR1 somewhat decreased cell growth inhibition and death in ATO-treated HPASMCs, which was accompanied by reduced oxidative stress.

\section{Introduction}

Vascular smooth muscle cells (VSMCs) are a dynamic constituent of the vasculature. Depending on pathological stimuli, VSMCs are involved in death, hypertrophy, or proliferation associated with hypertension, restenosis, and atherosclerosis $(1,2)$. Reactive oxygen species (ROS) control many cellular processes such as proliferation, migration, apoptosis, and secretion of inflammatory cytokines in VSMCs $(1,2)$. Lung tissues are easily injured by oxidative stress as they are exposed to an oxygen-rich environment. ROS in lung tissues are related to pulmonary vascular physiology. For instance, ROS regulate contractile ability by tuning an intracellular calcium level in human pulmonary artery smooth muscle cells (HPASMCs) (2). ROS are also involved in the development of pulmonary hypertension, ultimately inducing right ventricular failure and death (2). Hence, understanding the physiological or pathophysiological roles of ROS in the VSMCs of lung tissue are important.

Oxygen-derived molecules such as the hydroxyl radical $\left({ }^{\circ} \mathrm{OH}\right)$, superoxide anion $\left(\mathrm{O}_{2}{ }^{\circ}\right)$, and hydrogen peroxide $\left(\mathrm{H}_{2} \mathrm{O}_{2}\right)$ are principal cellular ROS (3). Cells have various antioxidants that function as redox rheostat, which is imperative for their survival and physiological events. The main metabolic antioxidant enzyme is superoxide dismutase (SOD), which processes $\mathrm{O}_{2}{ }^{--}$to $\mathrm{H}_{2} \mathrm{O}_{2}$ (4). Additional metabolism by catalase or glutathione (GSH) peroxidase produces stable molecules $\mathrm{O}_{2}$ and $\mathrm{H}_{2} \mathrm{O}$ (5). Thioredoxin ( $\mathrm{Trx}$ ) is a small antioxidant protein $(\sim 12 \mathrm{kDa})$ containing redox active cysteine residues at the active site (6). The oxidized disulfide form of Trx is reduced back to the reduced dithiol form by nicotinamide adenine dinucleotide phosphate (NADPH)-dependent Trx reductase (TrxR) (6). Thioredoxin 1 ( $\operatorname{Tr} x 1)$ and $\operatorname{Trx}$ reductase1 (TrxR1) are usually localized in the cytoplasm; however, $\operatorname{Trx} 2$ and $\operatorname{Trx} R 2$ are 
located in the mitochondria (6). Trx1 is implicated in cell growth, survival, tumor development, and angiogenesis (7-9). In addition, Trx is an anti-inflammatory and antioxidant cytokine involved in various inflammatory disorders, especially pulmonary diseases (10-12). Trx and TrxR can be inhibited by specific drugs. For example, PX-12 (1-methylpropyl 2-imidazolyl disulfide) is an irreversible Trx-1 inhibitor $(13,14)$ and auranofin is a TrxR inhibitor (14). Both PX-12 and auranofin exert antitumor effects in various cancer cells (15-20). Although these drugs do not perfectly and efficiently block the activities of Trx and TrxR, it is plausible to use PX-12 and auranofin to decrease the activities of Trx and TrxR in in vitro experiments.

Arsenic is a poison widely distributed throughout the environment and presents an international public health problem. Humans are exposed to arsenic mostly through ingestion of arsenic-contaminated water, food, and air (21). Conversely, inorganic arsenic has long been used to treat several severe diseases, and specifically, arsenic trioxide (ATO; $\mathrm{As}_{2} \mathrm{O}_{3}$ ) has recently been shown to be an effective therapeutic drug in certain leukemia patients $(21,22)$. ATO can disturb the natural oxidation and reduction equilibrium in cells by altering various redox enzymes $(23,24)$. ATO, as a mitochondrial toxin, induces a loss of mitochondrial membrane potential $(25,26)$ thereby increasing the production of ROS and consequently triggering apoptosis in target cells (27-29). Currently ATO is considered a TrxR inhibitor (30).

The development of numerous vascular diseases has been associated with chronic arsenic exposure in epidemiological studies (31-34). Blood vessel cells are the most susceptible to arsenic toxicity (31-34). Assimilation of arsenic for a prolonged period leads to pulmonary complications such as cough, bronchitis, and asthma (35). Recently, we reported that ATO induces growth inhibition and death in HPASMCs accompanied by mitochondrial $\mathrm{O}_{2}{ }^{--}$increase and GSH depletion (36). In the present study, the roles of Trx 1 and TrxR1 proteins in the regulation of cell growth, death, ROS, and GSH levels were investigated in ATO-treated HPASMCs, and the effects of PX-12 and auranofin on cell metabolism, ROS, and GSH levels in HPASMCs were examined.

\section{Materials and methods}

Cell culture. Primary HPASMCs obtained from PromoCell $\mathrm{GmbH}$ were maintained in a humidified incubator containing $5 \% \mathrm{CO}_{2}$ at $37^{\circ} \mathrm{C}$. HPASMCs were cultured in complete SMC Growth Medium 2 obtained from PromoCell GmbH. Cells were grown in 100-mm plastic tissue culture dishes (Nunc) and washed and detached with HepesBSS (30 mM HEPES), trypsin-EDTA, and trypsin neutralization solution (PromoCell $\mathrm{GmbH})$. For the experiments, HPASMCs between six and seven passages were used.

Reagents. ATO was purchased from Sigma-Aldrich/Merck $\mathrm{KGaA}$ and liquefied in $1.65 \mathrm{M} \mathrm{NaOH}$ at $10 \mathrm{mM}$. PX-12 was purchased from Tocris Bioscience and auranofin was obtained from Santa Cruz Biotechnology. Both drugs were dissolved in dimethyl sulfoxide (DMSO; Sigma-Aldrich; Merck KGaA) at $100 \mathrm{mM}$ as a stock solution.

Infection of cells with adLacZ, adTrx1, and adTrxR1. Overexpression of Trx1 and TrxR1 was accomplished using an adenoviral gene transfer. adLacZ, adTrx1 and adTrxR1 were provided by Dr J. Sadoshima (New Jersey Medical School, Newark, NJ, USA) and infection of cells was performed as previously described (37-39). Briefly, 7x10 10 cells in 6-well plates (Nunc) were incubated in RPMI-1640 medium (Thermo Fisher Scientific,Inc.) supplemented with $10 \%$ fetal bovine serum(FBS). Cells ( $70-80 \%$ confluence) in each well were infected with the same titers of control (CTR) adLacZ, adTrx1, or adTrxR1, which were determined using cell culture plague assays. One day later, cells were treated with or without $50 \mathrm{mM}$ ATO for an additional $24 \mathrm{~h}$. The infected cells were collected and used for western blot analysis, 3-(4,5-dimethylthiazol-2-yl)-2,5-diphenyltetrazolium bromide (MTT) assay, Annexin V-FITC staining, as well as ROS and GSH level measurements.

Transfection of cells with Trx1 and TrxR1 siRNAs. Gene silencing of Trx1 and TrxR1 was performed as previously described (37-39). A non-specific CTR siRNA duplex (5'-CCU ACGCCACCAAUUUCGU(dTdT)-3'), Trx1 siRNA duplex (5'-GCAUGCCAACAUUCCAGUU(dTdT)-3') and TrxR1 siRNA duplex (5'-GUCGUCUAUGAGAAUGCUU(dTdT)-3') were acquired from the Bioneer Corporation. In brief, $7 \times 10^{5}$ cells in 6-well plates (Nunc) were incubated in RPMI-1640 medium supplemented with $10 \%$ FBS. The next day, cells ( 70-80\% confluence) in each well were transfected with the CTR, Trx1, and TrxR1 siRNA duplex (80 pmol in Opti-MEM; Gibco; Thermo Fisher Scientific, Inc.) using Lipofectamine 2000 according to the manufacturer's instructions (Invitrogen; Thermo Fisher Scientific, Inc.). One day later, cells were treated with or without $50 \mathrm{mM}$ ATO. The transfected cells were used for MTT assays as well as ROS and GSH level measurements.

Western blot analysis. Protein expression levels of Trx1 and TrxR1 in HPASMCs were evaluated using western blotting. Briefly, cells were washed with PBS and lysed in 4 volumes of lysis buffer (Intron Biotechnology). Protein concentrations of clarified lysates were determined using the Bradford method. Protein samples (20 $\mu \mathrm{g}$ per well) were loaded and resolved on 4-15\% SDS-PAGE gels, and then transferred to Immobilon-P PVDF membranes (Merck Millipore) using electroblotting. Then, membranes were probed with anti-Trx1 (cat. no. sc-20146), anti-TrxR1 (cat. no. sc-28321) and anti- $\beta$-actin (cat. no. sc-47778) antibodies (1,000:1) (Santa Cruz Biotechnology). Membranes were incubated with horseradish peroxidase-conjugated secondary antibodies (cat. nos. sc-2357 or sc-2005) (1,000:1) (Santa Cruz Biotechnology). Blots were developed using an EZ-Western Lumi Pico ECL solution kit (DoGen).

Cell growth and metabolism assays. Alterations in cell growth and metabolism in the adenovirus-infected or siRNA-treated HPASMCs with or without ATO, PX-12, or auranofin treatment were indirectly determined by measuring the absorbance of MTT dye (Sigma-Aldrich; Merck KGaA) as previously described (38-41). The reduction of MTT dye to formazan by $\mathrm{NAD}(\mathrm{P}) \mathrm{H}$-dependent oxidoreductase enzymes depends on the cellular metabolic activity (41). In brief, $3 \times 10^{4}$ cells per well were seeded in 96-well microtiter plates (Nunc) for MTT assays. After exposure to the indicated amount of ATO, PX-12, or auranofin for 4 or $24 \mathrm{~h}, 20 \mu \mathrm{l}$ of MTT solution $(2 \mathrm{mg} / \mathrm{ml}$ in phosphate-buffered saline, PBS) was added to each well of the 
96-well plates (Nunc). The plates were additionally incubated for $\sim 3-4 \mathrm{~h}$ at $37^{\circ} \mathrm{C}$. The medium in the plates was withdrawn by pipetting and $100 \mu \mathrm{l}$ DMSO was added to each well to solubilize the formazan crystals. The optical concentration was measured at $570 \mathrm{~nm}$ using a microplate reader $\left(\right.$ Synergy $^{\mathrm{TM}}$ 2; BioTek Instruments Inc.).

Annexin V-FITC staining for the detection of apoptosis. Apoptosis was determined by staining cells with Annexin V-fluorescein isothiocyanate (FITC, Invitrogen; Thermo Fisher Scientific, Inc.; Ex/Em=488/519 nm) as previously described $(16,37)$. Adenovirus-infected HPASMCs were incubated with the indicated amount of ATO for $24 \mathrm{~h}$. Annexin V-FITC stained cells were analyzed with a FACStar flow cytometer (BD Biosciences).

Detection of intracellular ROS levels. ROS levels were measured using oxidation-sensitive fluorescent probe dyes, 2',7'-dichlorodihydrofluorescein diacetate $\left(\mathrm{H}_{2} \mathrm{DCFDA} ; \mathrm{Ex} / \mathrm{Em}=495 / 529 \mathrm{~nm}\right.$; Invitrogen; Thermo Fisher Scientific, Inc.) and dihydroethidium (DHE; Ex/Em=518/605 nm; Invitrogen; Thermo Fisher Scientific, Inc.). DHE is highly selective for $\mathrm{O}_{2}{ }^{--}$among ROS. In addition, the mitochondrial $\mathrm{O}_{2}^{-}$level was specifically detected using MitoSOX ${ }^{\mathrm{TM}}$ Red mitochondrial $\mathrm{O}_{2}{ }^{-}$indicator (Ex/Em=510/580 nm; Invitrogen; Thermo Fisher Scientific, Inc.). In brief, $1 \times 10^{6} \mathrm{HPASMCs} / \mathrm{ml}$ in a FACS tube (BD Biosciences) were incubated with $20 \mu \mathrm{M} \mathrm{H}_{2}$ DCFDA, DHE, or MitoSOX ${ }^{\mathrm{TM}}$ Red dye for 5, 30, 60, 120 or $180 \mathrm{~min}$. The $\mathrm{H}_{2} \mathrm{DCFDA}$, DHE, and MitoSOX ${ }^{\text {TM }}$ Red fluorescence levels were evaluated using a FACStar flow cytometer (BD Biosciences). The ROS levels were expressed as mean fluorescence intensity (MFI), which was calculated using CellQuest software (BD Biosciences). In addition, adenovirus-infected HPASMCs in the presence or absence of ATO for $24 \mathrm{~h}$ were incubated with $20 \mu \mathrm{M} \mathrm{H}_{2}$ DCFDA or DHE at $37^{\circ} \mathrm{C}$ for $30 \mathrm{~min} . \mathrm{H}_{2}$ DCFDA or DHE fluorescence was assessed using a FACStar flow cytometer (BD Biosciences) and the levels were expressed as MFI.

Detection of intracellular GSH levels. Cellular GSH levels were analyzed using a 5-chloromethylfluorescein diacetate dye (CMFDA, Ex/Em=522/595 nm; Invitrogen; Thermo Fisher Scientific, Inc.) as previously described (42). In brief, $1 \times 10^{6}$ HPASMCs $/ \mathrm{ml}$ in a FACS tube (BD Biosciences) were incubated with $5 \mu \mathrm{M}$ CMFDA for $5,30,60,120$ or $180 \mathrm{~min}$. The CMF fluorescence levels were evaluated using a FACStar flow cytometer (BD Biosciences). CMF (GSH) levels were expressed as MFI, which was calculated using CellQuest software (BD Biosciences).

Statistical analysis. Data are expressed as the mean of at least two independent experiments [mean \pm standard deviation (SD)]. The data were analyzed using Instat software (GraphPad Prism5; GraphPad Software, Inc.). The Student's t-test or one-way analysis of variance (ANOVA) with post hoc analysis using Tukey's multiple comparison test was performed for parametric data. A $\mathrm{P}$-value $<0.05$ was considered to indicate statistical significance.

\section{Results}

Effects of adTrxl and adTrxR1 on cell growth and death in ATO-treated HPASMCs. HPASMCs were infected with either
adLacZ, adTrx1, or adTrxR1 for the overexpression of Trx1 or TrxR1. In addition, $50 \mu \mathrm{M}$ ATO and $24 \mathrm{~h}$ were used as a suitable dose and time, respectively, due to the confluence of experimental cells. As shown in Fig. 1A, HPASMCs infected with adTrx1 or adTrxR1 exhibited increased Trx1 or TrxR1 protein levels compared with the cells infected with the control adLacZ. Based on MTT assays, $50 \mu \mathrm{M}$ ATO inhibited the growth of HPASMCs $\sim 25 \%$ at $24 \mathrm{~h}$ (Fig. 1B). Administration of adTrx1 slightly attenuated cell growth inhibition caused by ATO (Fig. 1B). In addition, adTrxR1 significantly reduced the inhibition of HPASMCs and metabolically increased the reduction of MTT to formazan in the control HPASMCs (Fig. 1B). When HPASMCs were stained with Annexin V-FITC to evaluate cell death, the percentage of Annexin V-stained cells was increased in the ATO-treated HPASMCs at $24 \mathrm{~h}$ (Fig. 1C). Both adTrx1 and adTrxR1 slightly prevented the death of ATO-treated HPASMCs (Fig. 1C).

Effects of adTrxl and adTrxRI on ROS and GSH levels in ATO-treated HPASMCs. To evaluate intracellular ROS levels in the ATO-treated HPASMCs, $\mathrm{H}_{2}$ DCFDA, DHE and MitoSOX ${ }^{\mathrm{TM}}$ Red dyes were used. Since ROS are short live radicals, ROS and GSH levels were assessed at the early time points of 5,30,60,120 or 180 min after ATO treatment. ATO increased ROS (DCF) levels at $5 \mathrm{~min}$ in the adLacZ, adTrx1, and adTrxR1-infected HPASMCs when compared with the untreated cells (Fig. 2A). Then, ATO decreased the ROS (DCF) levels to the level of the adLacZ-infected control cells (CTR adLacZ) at 30-180 min. Control HPASMCs infected with adTrx 1 or adTrxR1 showed a gradual decrease in ROS (DCF) levels at $30 \mathrm{~min}$ and the decreased levels persisted for $180 \mathrm{~min}$ (Fig. 2A). At $24 \mathrm{~h}$, ATO reduced ROS (DCF) levels in the HPASMCs (Fig. 2B). adTrx1 and adTrxR1 attenuated the reduced levels of ROS (DCF) in ATO-treated HPASMCs, and both adenoviruses did not significantly affect the ROS (DCF) levels which remained approximately similar as ROS levels in the control HPASMCs (adLacZ) (Fig. 2B).

Compared with the levels of adLacZ-infected control (CTR adLacZ) cells, ATO increased DHE $\left(\mathrm{O}_{2}^{\circ}\right)$ levels in the adLacZ, adTrx1, and adTrxR1-infected HPASMCs at 5 min (Fig. 3A). In addition, control cells infected with adTrx1 or adTrxR1 showed an increase in DHE $\left(\mathrm{O}_{2}^{\circ}\right)$ levels regardless of ATO at 5 min (Fig. 3A). Then, the ATO-increased DHE $\left(\mathrm{O}_{2}{ }^{-}\right)$levels decreased to the levels of adLacZ-infected control cells at 120-180 min (Fig. 3A). adTrx1 and adTrxR1 further decreased the DHE $\left(\mathrm{O}_{2}^{\circ}\right)$ levels in ATO-treated cells at $\sim 30-180 \mathrm{~min}$ and adTrx 1 reduced DHE $\left(\mathrm{O}_{2}^{-}\right)$levels in the control HPASMCs at $120 \mathrm{~min}$ (Fig. 3A). In addition, ATO increased mitochondrial $\mathrm{O}_{2}{ }^{-}$levels detected using MitoSOX ${ }^{\mathrm{TM}}$ Red dye at 5 min whereas adTrx1 and adTrxR1 attenuated the increased levels in these cells (Fig. 3B). adTrx 1 and adTrxR1 decreased mitochondrial $\mathrm{O}_{2}^{-{ }^{-}}$levels in the control HPASMCs at $\sim 30-180 \mathrm{~min}$ (Fig. 3B). At $24 \mathrm{~h}$, ATO significantly increased DHE $\left(\mathrm{O}_{2}^{\circ}\right)$ levels in the control HPASMCs (Fig. 3C). Although adTrx 1 attenuated the increased DHE $\left(\mathrm{O}_{2}{ }^{\circ}\right)$ levels in ATO-treated cells, the levels were not significantly changed by adTrx R1 (Fig. 3C). In addition, adTrx1 and adTrxR1 did not alter the basal level of DHE $\left(\mathrm{O}_{2}{ }^{-}\right)$ in the control HPASMCs (Fig. 3C).

When intracellular GSH levels were measured in adenovirus-infected HPASMCs using a CMFDA dye, $50 \mu \mathrm{M}$ ATO 
A

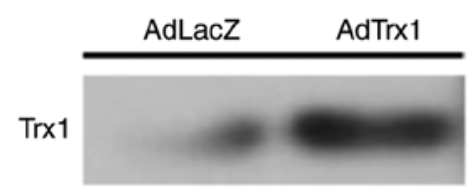

$\beta$-actin

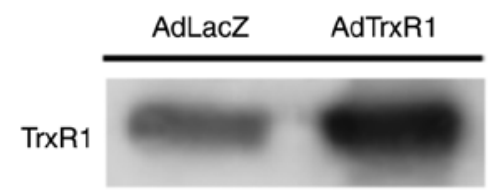

$\beta$-actin

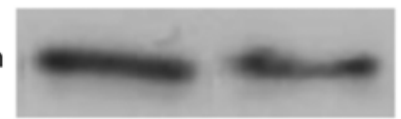

C
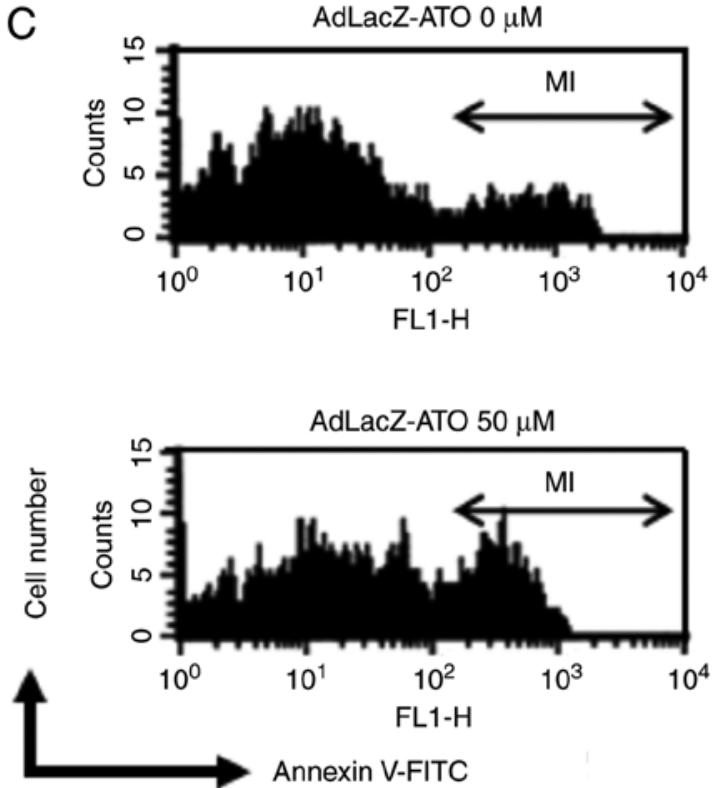
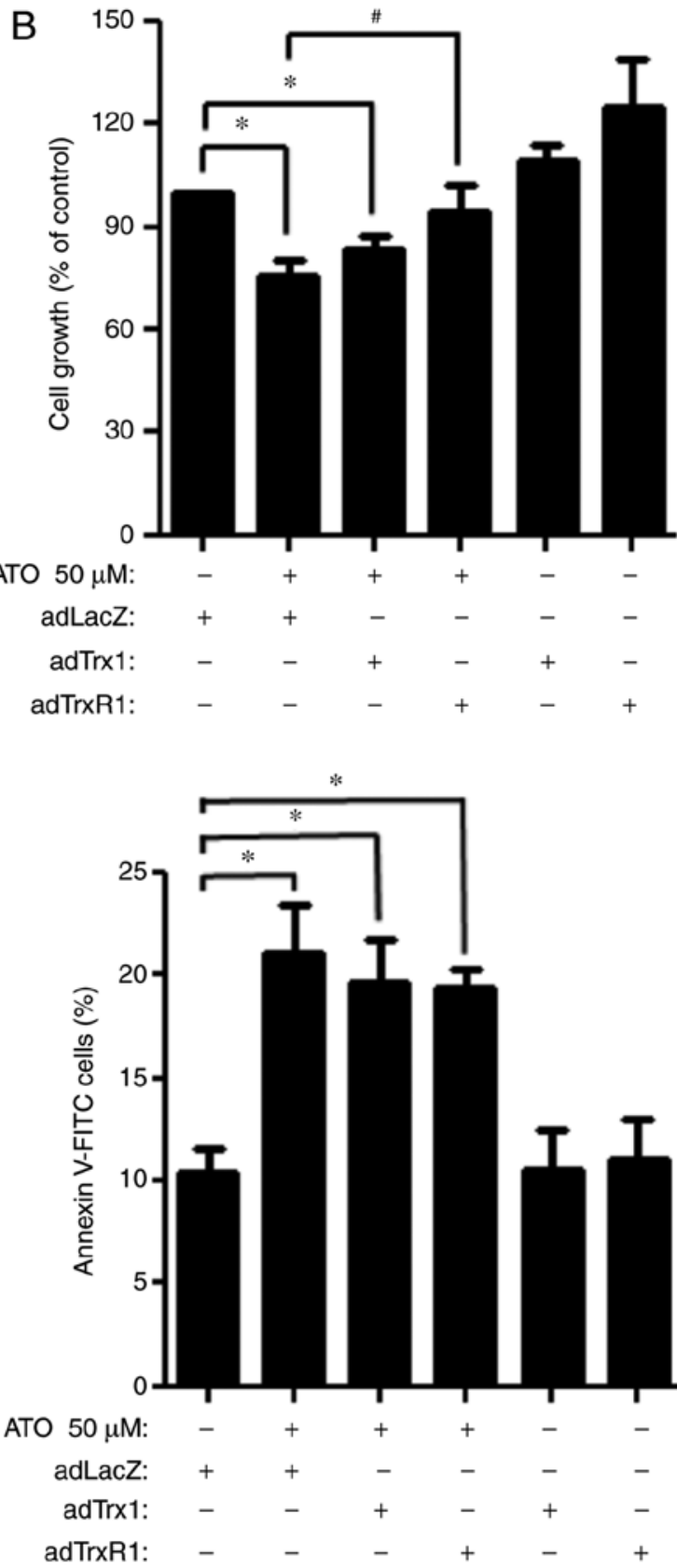

Figure 1. Effects of adTrx1 and adTrxR1 on cell growth and death in HPASMCs. HPASMCs ( 70-80\% confluence) were infected with control adLacZ, adTrx1 or adTrxR1. Twenty-four hours later, the cells were treated with $50 \mu \mathrm{M}$ ATO for an additional 24 h. (A) Expression levels of Trx1 and TrxR1 proteins in HPASMCs. (B) Cellular growth changes assessed by the reduction of MTT to formazan for $4 \mathrm{~h}$. (C) Representative images (left) of Annexin V-FITC staining cells, as analyzed by FACStar flow cytometer. M1 regions show the Annexin V-FITC-positive cells. Graph (right) indicates the percentages of Annexin V-FITC-positive cells. ${ }^{*} \mathrm{P}<0.05$ compared with the adLacZ-infected control group; ${ }^{\#} \mathrm{P}<0.05$ compared with the adLacZ-infected control cells treated with ATO only. Trx1, thioredoxin 1; TrxR1, Trx reductase1; ATO, arsenic trioxide $\left(\mathrm{As}_{2} \mathrm{O}_{3}\right)$; HPASMCs, human pulmonary artery smooth muscle cells.

significantly increased GSH (CMF) levels at 5 min and then gradually decreased to the control levels at $180 \mathrm{~min}$ (Fig. 4). adTrx1 and adTrxR1 reduced the increased GSH (CMF) levels in ATO-treated cells at 5-60 min (Fig. 4). In addition, adTrx1 and adTrxR1 decreased GSH (CMF) levels in the control HPASMCs at 5-30 min (Fig. 4).

Effects of Trxl and TrxR1 siRNA on cell metabolism, ROS, and GSH levels in ATO-treated HPASMCs. Next, HPASMCs were transfected with either control, Trx1 siRNA or TrxR1
siRNA for downregulation of the corresponding proteins. As shown in Fig. 5A, Trx1 siRNA suppressed Trx1 expression in the HPASMCs, and TrxR1 siRNA also downregulated the expression of TrxR1 protein. ATO decreased the reduction of MTT to formazan in the control siRNA (CTR siRNA)-treated HPASMCs at $4 \mathrm{~h}$. The metabolism of HPASMCs transfected with Trx1 or TrxR1 siRNA was significantly reduced regardless of ATO (Fig. 5B).

Regarding ROS and GSH levels, HPASMCs treated with Trx1 or TrxR1 siRNA showed increased ROS (DCF) levels 

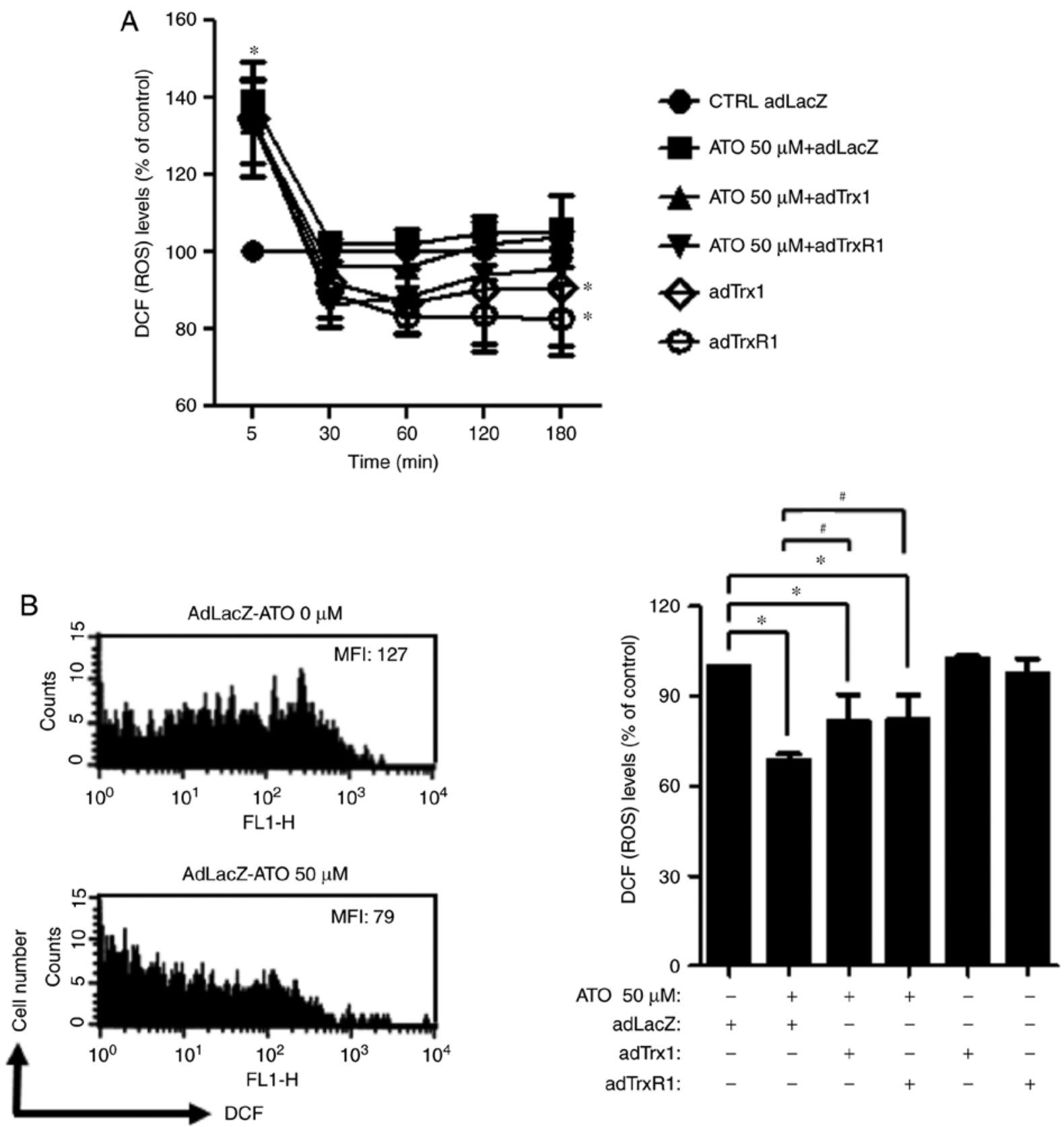

Figure 2. Effects of adTrx1 and adTrxR1 on ROS levels in HPASMCs. HPASMCs ( 70-80\% confluence) were infected with CTR adLacZ, adTrx1 or adTrxR1. Twenty-four hours later, the cells were treated with $50 \mu \mathrm{M}$ ATO for the indicated times. ROS levels in HPASMCs were measured using a FACStar flow cytometer. (A) The graph indicate DCF (ROS) levels (\% of adLacZ-infected control cells) at the indicated times. (B) Representative images (left) of DCF-staining cells. MFI, mean fluorescence intensity. The graph (right) indicate DCF (ROS) levels (\% of adLacZ-infected control cells) at $24 \mathrm{~h}$. "P<0.05 compared with the adLacZ-infected control group; ${ }^{\prime} \mathrm{P}<0.05$ compared with the adLacZ-infected control cells treated with ATO only. Trx1, thioredoxin 1; TrxR1, Trx reductase1; ATO, arsenic trioxide $\left(\mathrm{As}_{2} \mathrm{O}_{3}\right)$; HPASMCs, human pulmonary artery smooth muscle cells; ROS, reactive oxygen species.

with or without ATO at 5 min (Fig. 5C). In particular, TrxR1 siRNA-treated cells had higher ROS (DCF) levels (Fig. 5C). Then, the increased ROS (DCF) levels returned to the levels of control siRNA-treated cells at $\sim 30-180 \mathrm{~min}$ (Fig. 5C). Trx1 and TrxR1 siRNAs, specifically Trx1 siRNA, reduced the increased GSH (CMF) levels in the ATO-treated cells at $\sim 5-180 \mathrm{~min}$ (Fig. 5D). Comparatively, Trx1 and TrxR1 siRNAs decreased GSH (CMF) levels in the control HPASMCs at $\sim 5-30$ min (Fig. 5D).

Effects of PX-12 and auranofin on cell metabolism, ROS, and GSH levels in HPASMCs. Treatment with $30 \mu \mathrm{M}$ PX-12 and $5 \mu \mathrm{M}$ auranofin decreased the cellular metabolism of
HPASMCs $\sim 40 \%$ at $4 \mathrm{~h}$ compared with the untreated cells (Fig. 6A). Furthermore, $100 \mu \mathrm{M}$ PX-12 and $30 \mu \mathrm{M}$ auranofin nearly ceased the activity of cellular metabolism, indicating that the cells were gradually dying (Fig. 6A). Regarding ROS and GSH levels, $100 \mu \mathrm{M}$ PX-12 and $30 \mu \mathrm{M}$ auranofin increased ROS (DCF) levels in HPASMCs at $~ 30-180$ min compared with the control cell (CTR) levels (Fig. 6B). HPASMCs treated with $30 \mu \mathrm{M}$ PX-12 and $5 \mu \mathrm{M}$ auranofin showed increased ROS (DCF) levels at $\sim 120-180$ min (Fig. 6B). The tested doses of PX-12 and auranofin reduced GSH (CMF) levels in HPASMCs at $30 \mathrm{~min}$ and the decreased levels returned to $90 \%$ of the control GSH (CMF) levels at 180 min (Fig. 6C). 

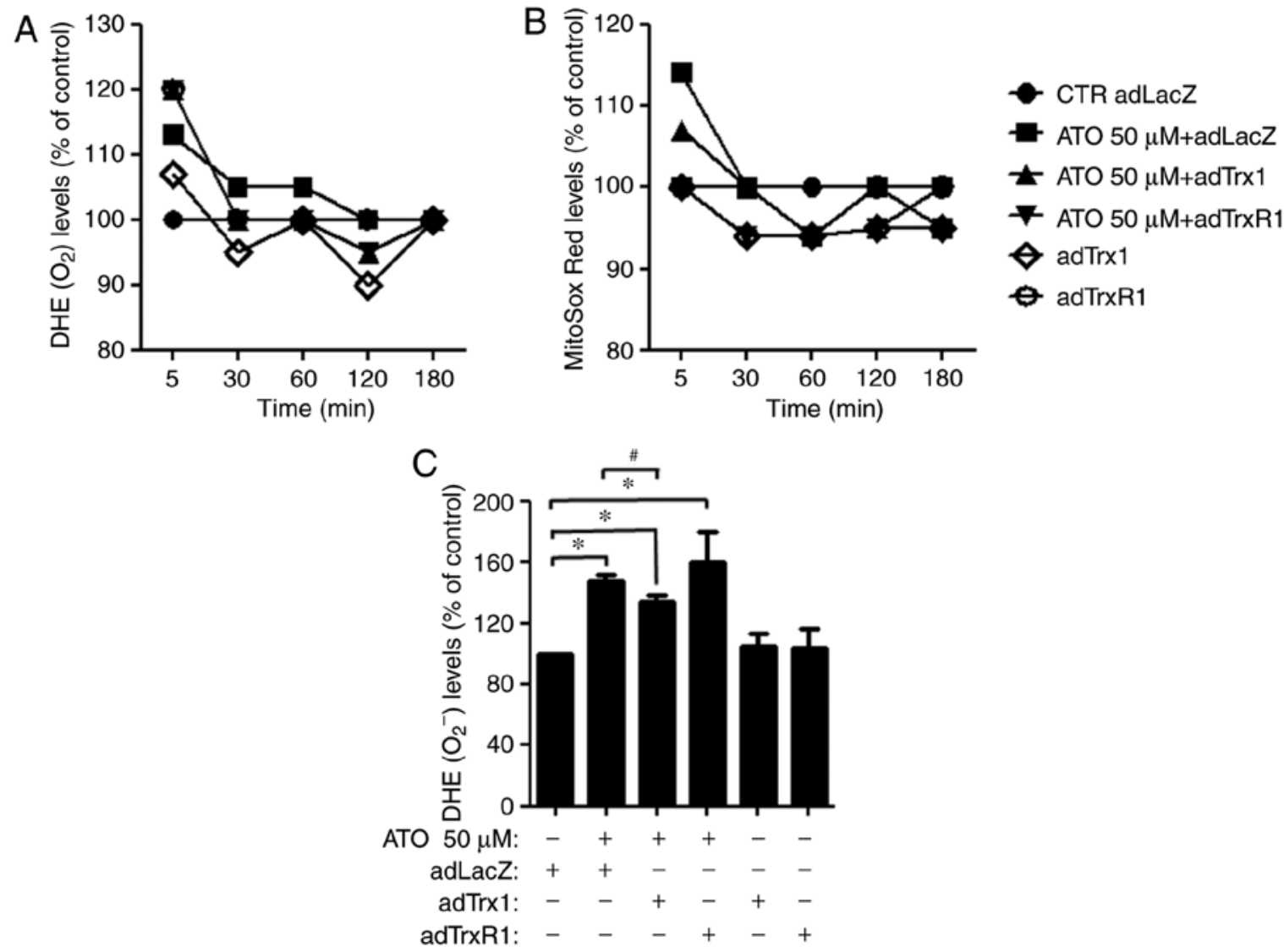

Figure 3. Effects of adTrx1 and adTrxR1 on $\mathrm{O}_{2}^{-*}$ levels in HPASMCs. HPASMCs ( 70-80\% confluence) were infected with CTR adLacZ, adTrx1, or adTrxR1. Twenty-four hours later, the cells were treated with $50 \mu \mathrm{M}$ ATO for the indicated times. DHE $\left(\mathrm{O}_{2}^{\circ}\right)$ and MitoSOX ${ }^{\mathrm{TM}} \operatorname{Red}\left(\operatorname{mitochondrial} \mathrm{O}_{2}{ }^{\circ}\right)$ levels in HPASMCs were measured using a FACStar flow cytometer. (A) DHE $\left(\mathrm{O}_{2}^{*}\right)$ and (B) MitoSOX ${ }^{\mathrm{TM}}$ Red (mitochondrial $\mathrm{O}_{2}^{*}$ ) levels compared with the adLacZ-infected control cells at the indicated early times. (C) DHE $\left(\mathrm{O}_{2}^{\circ}\right)$ levels (\% of adLacZ-infected control cells) at $24 \mathrm{~h}$. ${ }^{*} \mathrm{P}<0.05$ compared with the adLacZ-infected control group; ${ }^{\#} \mathrm{P}<0.05$ compared with the adLacZ-infected control cells treated with ATO only. Trx 1, thioredoxin 1; TrxR1, Trx reductase1; ATO, arsenic trioxide $\left(\mathrm{As}_{2} \mathrm{O}_{3}\right)$; HPASMCs, human pulmonary artery smooth muscle cells; DHE, dihydroethidium.

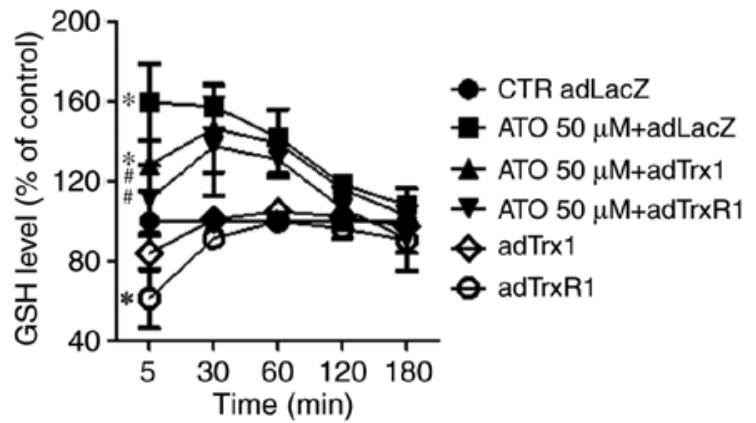

Figure 4. Effects of adTrx1 and adTrxR1 on GSH levels in HPASMCs. HPASMCs ( 70-80\% confluence) were infected with CTR adLacZ, adTrx1, or adTrxR1. Twenty-four hours later, the cells were treated with $50 \mu \mathrm{M}$ ATO for the indicated times. GSH levels in HPASMCs were measured using a FACStar flow cytometer. The graph indicates GSH (CMF) levels compared with the adLacZ-infected control cells at the indicated early times. ${ }^{*} \mathrm{P}<0.05$ compared with the adLacZ-infected control group. ${ }^{\text {}} \mathrm{P}<0.05$ compared with the adLacZ-infected control cells treated with ATO only. Trx1, thioredoxin 1; TrxR1, Trx reductase1; ATO, arsenic trioxide $\left(\mathrm{As}_{2} \mathrm{O}_{3}\right)$; HPASMCs, human pulmonary artery smooth muscle cells; GSH, glutathione.

\section{Discussion}

The thioredoxin (Trx) system consists of $\operatorname{Trx}$, TrxR, and $\mathrm{NADPH}$, an important enzymatic complex that maintains the homeostasis of cellular redox (43). Specifically, thioredoxin $1(\operatorname{Trx} 1)$ and $\operatorname{Trx}$ reductase1 $(\operatorname{Trx} \mathrm{R} 1)$ is overexpressed in asthma, acute lung injury, and lung cancer $(11,37,44,45)$. Therefore, the Trx system can be a promising target for the treatment of pulmonary diseases. Recently, it was demonstrated that an environmental agent, arsenic trioxide (ATO), leads to growth inhibition and death in human pulmonary artery smooth muscle cells (HPASMCs) accompanied by mitochondrial $\mathrm{O}_{2}{ }^{--}$increase and GSH depletion (36). In addition, PX-12 and auranofin induce the death of lung cancer and mesothelioma cells through oxidative stress $(16,19)$. In the present study, whether changes in $\operatorname{Tr} x 1$ and $\operatorname{Tr} x \operatorname{R} 1$ expression affect cell growth, death, ROS, and GSH levels in ATO-treated HPASMCs was investigated and the effects of PX-12 and auranofin on cell metabolism and redox status in HPASMCs was analyzed.

HPASMCs infected with adTrx 1 and adTrxR 1 showed upregulation of Trx1 and TrxR1 proteins. Overexpression of Trx1 and TrxR1, especially TrxR1, attenuated cell growth inhibition caused by ATO. Both adenoviruses moderately prohibited the death of ATO-treated HPASMCs. In our previous studies, adTrx1 was also shown to reduce cell death in suberoylanilide hydroxamic or suberoyl bishydroxamic acid-treated lung and cervical cancer cells $(37-39,46)$. These results support that $\operatorname{Trx} 1$ and $\operatorname{TrxR} 1$ have anti-apoptotic and 

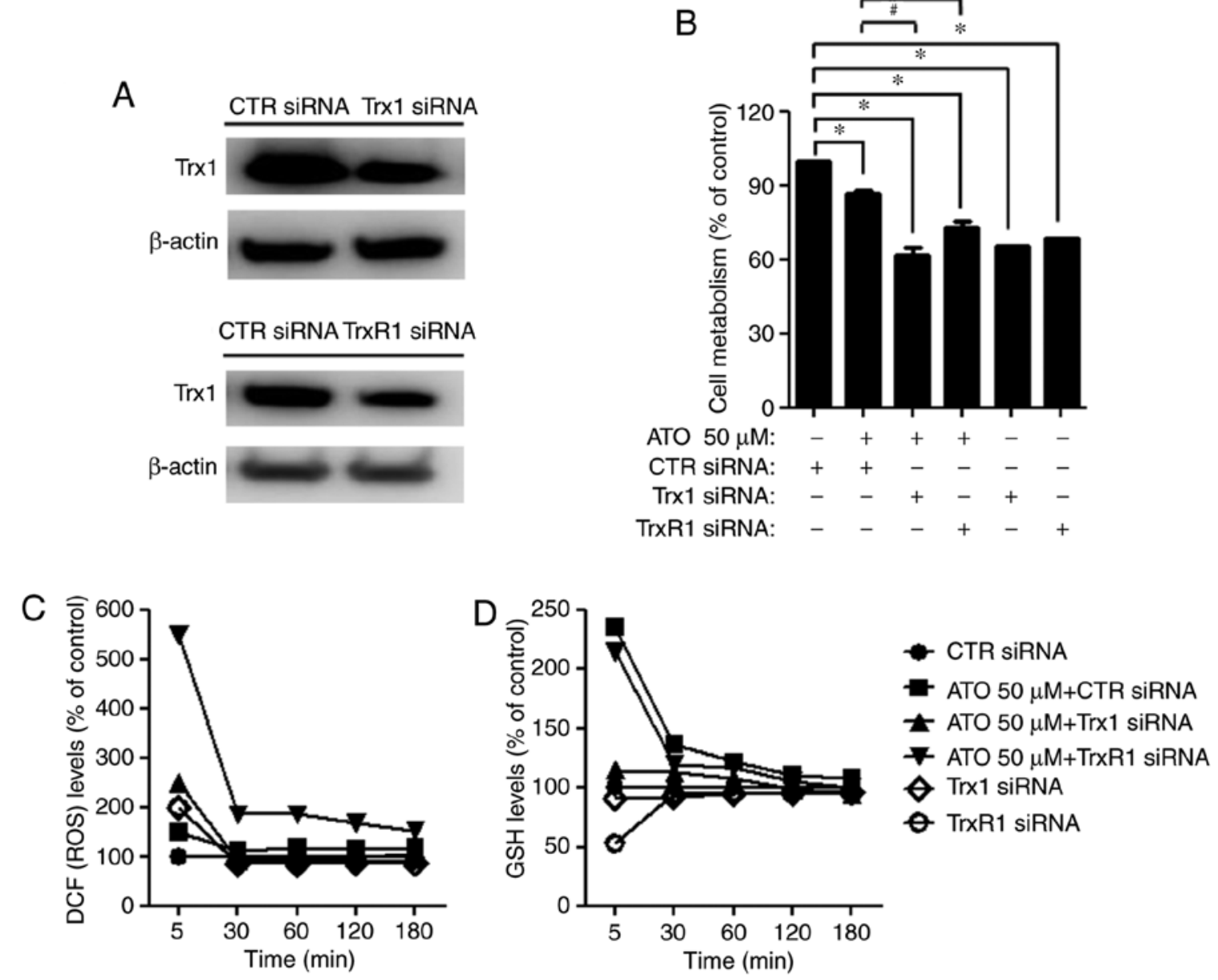

Figure 5. Effects of Trx1 siRNA and TrxR1 siRNA on cell metabolism, ROS, and GSH levels in HPASMCs. HPASMCs ( 70-80\% confluence) were transfected with CTR siRNA, Trx1 siRNA, or TrxR1 siRNA. Twenty-four hours later, the cells were treated with $50 \mu \mathrm{M}$ ATO for the indicated times. (A) Expression levels of Trx1 and TrxR1 proteins in HPASMCs. (B) Cellular metabolism changes measured by the reduction of MTT to formazan for $4 \mathrm{~h}$. (C and D) ROS and GSH levels in HPASMCs were measured using a FACStar flow cytometer. DCF (ROS) (C) and GSH (CMF) levels (D) compared with the control siRNA-treated cells at the indicated times. ${ }^{\text {P }} \mathrm{P}<0.05$ compared with the control siRNA-treated cells; ${ }^{*} \mathrm{P}<0.05$ compared with the control siRNA-treated cells treated with ATO only. Trx1, thioredoxin 1; TrxR1, Trx reductase1; ATO, arsenic trioxide $\left(\mathrm{As}_{2} \mathrm{O}_{3}\right)$; HPASMCs, human pulmonary artery smooth muscle cells; GSH, glutathione; ROS, reactive oxygen species.

cell survival effects (8), which may be important for cellular resistance to toxic agents. ATO diminished the reduction of MTT to formazan in HPASMCs, implying that ATO decreased the cellular metabolism of HPASMCs, consequently reducing the activity of NAD(P)H-dependent oxidoreductase. adTrxR1 efficiently increased cellular metabolism, indicated by the reduction of MTT to formazan in HPASMCs. In contrast, HPASMCs transfected with Trx1 or TrxR1 siRNA showed significant reduction of HPASMC metabolism regardless of ATO. In addition, PX-12 and auranofin, which presumably inhibited the activities of Trx1 and TrxR1, respectively, decreased the cellular metabolism of HPASMCs. Relatively higher doses of PX-12 and auranofin almost terminated the cellular metabolism at $4 \mathrm{~h}$, indicating that the cells were progressively dying. Since Trx can control numerous cell signaling pathways by modulating cell-type-specific transcription factors such as p38, NF- $\mathrm{B}, \mathrm{AP}-1$, p53, and nuclear receptors $(11,47-49)$, Trx is possibly involved in the regulation of basal cellular metabolism in glycolysis, the TCA cycle, and mitochondria to produce ATP or NAD $(\mathrm{P}) \mathrm{H}(50)$. However, these effects mediated by the Trx system may depend on cell type, co-treatment with drugs as well as basal activity and the level of Trx-related proteins.

ATO increases the production of ROS in numerous cells including SMCs (27-29,36). Likewise, ATO increased $\mathrm{O}_{2}{ }^{-}$levels at $24 \mathrm{~h}$ in HPASMCs. ATO also increased DCF (ROS) levels including the mitochondrial $\mathrm{O}_{2}{ }^{--}$at $5 \mathrm{~min}$. ATO apparently impaired mitochondria, leading to the generation of $\mathrm{O}_{2}{ }^{--}$, reduction of cell metabolism, and consequent induction of cell death. Mitochondria are the primary energetic subcellular organelles for generating $\mathrm{ROS}$ and have $\mathrm{O}_{2}{ }^{--}$content approximately 5-10-fold higher than that of the cytoplasm (51). However, at approximately 30-180 min, ATO did not increase ROS levels, including $\mathrm{O}_{2}^{-}$, compared with the control group cells. Moreover, ATO decreased ROS (DCF) levels at $24 \mathrm{~h}$. It is possible that the fluorescent DCF was not exactly detected due to the cell leakage of the dye and/or the deficiency in the esterase to remove the acetate groups from $\mathrm{H}_{2} \mathrm{DCFDA}$. ATO regulates ROS levels using a variety of redox enzymes $(23,24,52)$ and by causing mitochondrial dysfunction $(24,25,53)$. Therefore, ATO might change ROS levels in HPASMCs differently depending on incubation time and dose. In addition, a relatively low dose 


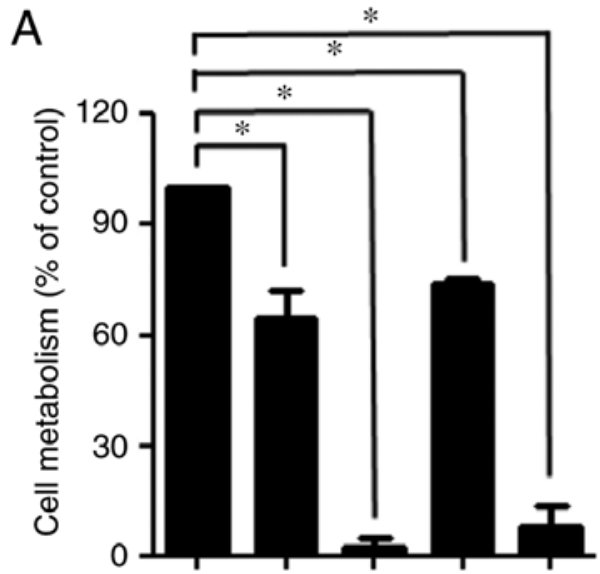

$\mathrm{PX}-12(\mu \mathrm{M}): \quad 0 \quad 30 \quad 100$ Auranofin $(\mu \mathrm{M}): \quad 0 \quad 5 \quad 30$
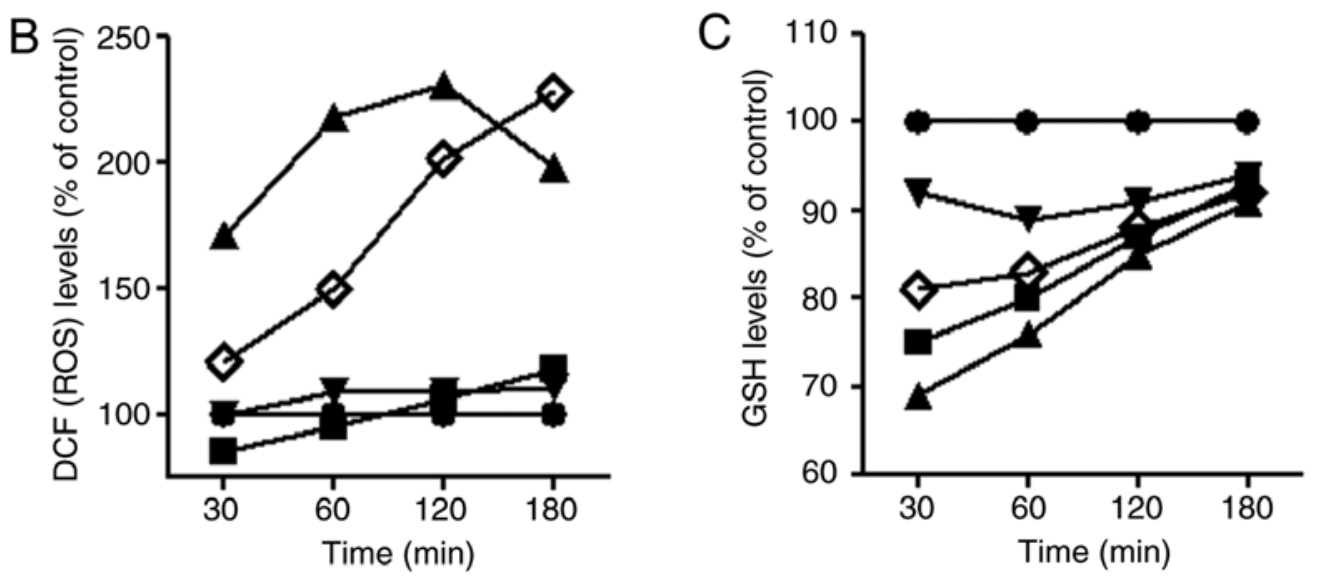

Figure 6. Effects of PX-12 and auranofin on cell metabolism, ROS, and GSH levels in HPASMCs. Approximately 90\% growing cells were treated with the indicated amounts of PX-12 and Auranofin for the indicated times. (A) Cellular metabolism changes measured by the reduction of MTT to formazan for $4 \mathrm{~h}$. (B and C) ROS and GSH levels in HPASMCs were measured using a FACStar flow cytometer. DCF (ROS) (B) and GSH (CMF) levels (C) compared with the CTR cells at the indicated times. "P<0.05 compared with the control group. HPASMCs, human pulmonary artery smooth muscle cells; GSH, glutathione; ROS, reactive oxygen species.

of ATO induces a significant oxidative stress as well as a time-dependent differential modulation of antioxidant status in a tissue-specific manner $(52,54)$. Oxidative damage markers such as protein nitration and lipid peroxidation could be very useful to discuss these results in depth. Moreover, if rotenone as a mitochondrial inhibitor were used and the mitochondria were isolated from HPASMCs to study metabolic activity, the results would be more informative.

Administration of $\operatorname{adTrx} 1$ or $\operatorname{adTrx} \mathrm{R} 1$ reduced the increased mitochondrial $\mathrm{O}_{2}^{--}$levels in ATO-treated HPASMCs at $5 \mathrm{~min}$. In addition, adTrx 1 and adTrxR 1 decreased the mitochondrial $\mathrm{O}_{2}^{--}$levels in the control HPASMCs at approximately 30-180 min. However, neither adenovirus decreased DCF (ROS) and DHE $\left(\mathrm{O}_{2}{ }^{-}\right)$levels in ATO-treated HPASMCs at $5 \mathrm{~min}$. The altered redox status via overexpression of $\operatorname{Tr} x$ primarily lead to the maintenance of mitochondrial integrity. Control HPASMCs infected with adTrx1 or adTrxR1 showed a steady decrease in ROS (DCF) levels at approximately 30-180 min. Furthermore, adTrx1 decreased DHE $\left(\mathrm{O}_{2}{ }^{\circ}\right)$ levels in ATO-treated HPASMCs at $24 \mathrm{~h}$ and adTrx 1 reduced DHE $\left(\mathrm{O}_{2}{ }^{-}\right)$levels in the control HPASMCs at $120 \mathrm{~min}$. In addi- tion, HPASMCs treated with Trx1 or TrxR1 siRNA showed increased ROS (DCF) levels with or without ATO at $5 \mathrm{~min}$. In particular, TrxR1 siRNA-treated cells showed higher ROS (DCF) levels. PX-12 and auranofin increased ROS (DCF) levels in HPASMCs. Although a minimal difference in ROS levels was observed between adTrx1- and ad TrxR1-treated cells, overexpression of Trx generally reduced ROS levels and downregulation of Trx increased ROS levels in HPASMCs. adTrx 1 and adTrxR1 did not augment the reduced ROS (DCF) levels in ATO-treated HPASMCs at $24 \mathrm{~h}$; overexpression of Trx most likely increased the basal ROS level by increasing cellular metabolism in these cells.

GSH is a non-protein thiol antioxidant crucial for protecting cells from oxidative stress and has a significant effect on ATO-, PX-12-, and auranofin-induced apoptosis in various cells including HPASMCs (16-20,28,36,55-57). ATO significantly increased GSH (CMF) levels at $5 \mathrm{~min}$ and then steadily reduced the levels at $180 \mathrm{~min}$. The short-lived increase of GSH levels was likely due to the defense mechanism against the transiently increased ROS levels caused by ATO. The subsequent decrease of GSH levels was possibly due to its use to decrease 
ROS levels. Trx1 and TrxR1 siRNAs decreased GSH (CMF) levels in the control HPASMCs at approximately 5-30 min and both siRNAs reduced the increased GSH (CMF) levels in ATO-treated cells at approximately 5-180 min. Dysregulation of the Trx system by Trx1 and TrxR1 siRNAs failed to produce the basal metabolic reducing agent of $\mathrm{NAD}(\mathrm{P}) \mathrm{H}$ to generate the reduced form of GSH, consequently decreasing GSH levels in these cells. In addition, PX-12 and auranofin reduced GSH (CMF) levels in HPASMCs at $30 \mathrm{~min}$, indicating that downregulation of the Trx system by siRNA or drugs diminishes the basal cellular metabolism and causes a cellular milieu of comparative oxidative stress. adTrx 1 and adTrxR1 attenuated the increased GSH (CMF) levels in ATO-treated cells at approximately 5-60 $\mathrm{min}$. In addition, both adenoviruses lowered the basal GSH (CMF) levels in HPASMCs at approximately 5-30 min. The upregulated activity of the Trx system via adTrx 1 and adTrxR1 possibly utilized NADPH during these time periods, which could not renew or create the reduced form of GSH, consequently decreasing GSH levels in these cells.

In summary, upregulation of Trx 1 and TrxR1 partially decreased growth inhibition and death in ATO-treated HPASMCs. Generally, overexpression of Trx reduced ROS levels in ATO-treated HPASMCs whereas downregulation of Trx increased ROS levels and decreased GSH levels in these cells. adTrxR1 seemed to efficiently increase cellular metabolism whereas Trx 1 or TrxR1 siRNA significantly decreased the cellular metabolism. Both PX-12 and auranofin decreased the cellular metabolism accompanied by an increase in ROS level and a decrease in GSH level. The results from the present study may contribute to research focusing on the Trx system for the treatment or prevention of pulmonary vascular diseases caused by contact with arsenic. Further characterizations of Trx and Trx R in vascular system should be investigated in future using alterative cells due to the difficulty working with primary normal HPASMCs.

\section{Acknowledgements}

Not applicable.

\section{Funding}

The present study was supported by the Basic Science Research Program through the National Research Foundation of Korea (NRF) funded by the Ministry of Education (2019R1I1A2A01041209).

\section{Availability of data and materials}

The datasets used during the present study are available from the corresponding author upon reasonable request.

\section{Authors' contributions}

WHP is the sole author of the study responsible for the design, experimental procedures, data collection and analysis and writing of the manuscript. WHP agrees to be accountable for all aspects of the research in ensuring that the accuracy and integrity of any part of the work are appropriately investigated and resolved.

\section{Ethics approval and consent to participate}

Not applicable.

\section{Patient consent for publication}

Not applicable.

\section{Competing interests}

The author declares no competing interests.

\section{References}

1. Irani K: Oxidant signaling in vascular cell growth, death, and survival: A review of the roles of reactive oxygen species in smooth muscle and endothelial cell mitogenic and apoptotic signaling. Circ Res 87: 179-183, 2000.

2. Perez-Vizcaino F, Cogolludo A and Moreno L: Reactive oxygen species signaling in pulmonary vascular smooth muscle. Respir Physiol Neurobiol 174: 212-220, 2010.

3. Trachootham D, Alexandre J and Huang P: Targeting cancer cells by ROS-mediated mechanisms: A radical therapeutic approach? Nat Rev Drug Discov 8: 579-591, 2009.

4. Zelko IN, Mariani TJ and Folz RJ: Superoxide dismutase multigene family: A comparison of the CuZn-SOD (SOD1), Mn-SOD (SOD2), and EC-SOD (SOD3) gene structures, evolution, and expression. Free Radic Biol Med 33: 337-349, 2002.

5. Wilcox CS: Reactive oxygen species: Roles in blood pressure and kidney function. Curr Hypertens Rep 4: 160-166, 2002.

6. Collet JF and Messens J: Structure, function, and mechanism of thioredoxin proteins. Antioxid Redox Signal 13: 1205-1216, 2010.

7. Lim JY, Yoon SO, Hong SW, Kim JW, Choi SH and Cho JY: Thioredoxin and thioredoxin-interacting protein as prognostic markers for gastric cancer recurrence. World J Gastroenterol 18: 5581-5588, 2012.

8. Pramanik KC and Srivastava SK: Apoptosis signal-regulating kinase 1-thioredoxin complex dissociation by capsaicin causes pancreatic tumor growth suppression by inducing apoptosis. Antioxid Redox Signal 17: 1417-1432, 2012.

9. Dunn LL, Buckle AM, Cooke JP and Ng MK: The emerging role of the thioredoxin system in angiogenesis. Arterioscler Thromb Vasc Biol 30: 2089-2098, 2010.

10. Tipple TE: The thioredoxin system in neonatal lung disease. Antioxid Redox Signal 21: 1916-1925, 2014.

11. $\mathrm{Xu} \mathrm{J}, \mathrm{Li} \mathrm{T}, \mathrm{Wu} \mathrm{H}$ and $\mathrm{Xu}$ T: Role of thioredoxin in lung disease. Pulm Pharmacol Ther 25: 154-162, 2012.

12. Nakamura T, Nakamura H, Hoshino T, Ueda S, Wada $H$ and Yodoi J: Redox regulation of lung inflammation by thioredoxin. Antioxid Redox Signal 7: 60-71, 2005.

13. Galmarini CM: Drug evaluation: The thioredoxin inhibitor PX-12 in the treatment of cancer. Curr Opin Investig Drugs 7: $1108-1115,2006$

14. May HC, Yu JJ, Guentzel MN, Chambers JP, Cap AP and Arulanandam BP: Repurposing Auranofin, Ebselen, and PX-12 as antimicrobial agents targeting the thioredoxin system. Front Microbiol 9: 336, 2018.

15. Wondrak GT: Redox-directed cancer therapeutics: Molecular mechanisms and opportunities. Antioxid Redox Signal 11: 3013-3069, 2009.

16. You BR, Shin HR, Han BR and Park WH: PX-12 induces apoptosis in Calu- 6 cells in an oxidative stress-dependent manner. Tumour Biol 36: 2087-2095, 2015.

17. You BR, Shin HR and Park WH: PX-12 inhibits the growth of A549 lung cancer cells via G2/M phase arrest and ROS-dependent apoptosis. Int J Oncol 44: 301-308, 2014.

18. Shin HR, You BR and Park WH: PX-12-induced HeLa cell death is associated with oxidative stress and GSH depletion. Oncol Lett 6: 1804-1810, 2013.

19. You BR and Park WH: Auranofin induces mesothelioma cell death through oxidative stress and GSH depletion. Oncol Rep 35: 546-551, 2016. 
20. You BR, Shin HR, Han BR, Kim SH and Park WH: Auranofin induces apoptosis and necrosis in HeLa cells via oxidative stress and glutathione depletion. Mol Med Rep 11: 1428-1434, 2015.

21. Khairul I, Wang QQ, Jiang YH, Wang C and Naranmandura $H$ : Metabolism, toxicity and anticancer activities of arsenic compounds. Oncotarget 8: 23905-23926, 2017.

22. Waxman S and Anderson KC: History of the development of arsenic derivatives in cancer therapy. Oncologist (6 Suppl 2): S3-S10, 2001.

23. Chou WC, Jie C, Kenedy AA, Jones RJ, Trush MA and Dang CV: Role of NADPH oxidase in arsenic-induced reactive oxygen species formation and cytotoxicity in myeloid leukemia cells. Proc Natl Acad Sci USA 101: 4578-4583, 2004.

24. Miller WH Jr, Schipper HM, Lee JS, Singer J and Waxman S: Mechanisms of action of arsenic trioxide. Cancer Res 62 3893-3903, 2002.

25. Park WH, Seol JG, Kim ES, Hyun JM, Jung CW, Lee CC, Kim BK and Lee YY: Arsenic trioxide-mediated growth inhibition in MC/CAR myeloma cells via cell cycle arrest in association with induction of cyclin-dependent kinase inhibitor, p21, and apoptosis. Cancer Res 60: 3065-3071, 2000.

26. Zhang W, Liu Y, An Z, Huang D, Qi Y and Zhang Y: Mediating effect of ROS on mtDNA damage and low ATP content induced by arsenic trioxide in mouse oocytes. Toxicol In Vitro 25 979-984, 2011.

27. Li JX, Shen YQ, Cai BZ, Zhao J, Bai X, Lu YJ and Li XQ: Arsenic trioxide induces the apoptosis in vascular smooth muscle cells via increasing intracellular calcium and ROS formation. Mol Biol Rep 37: 1569-1576, 2010.

28. You BR and Park WH: Arsenic trioxide induces human pulmonary fibroblast cell death via increasing ROS levels and GSH depletion. Oncol Rep 28: 749-757, 2012.

29. Cha Y, Park DW, Lee CH, Baek SH, Kim SY, Kim JR and Kim JH: Arsenic trioxide induces apoptosis in human colorectal adenocarcinoma HT-29 cells through ROS. Cancer Res Treat 38: 54-60, 2006

30. Lu J, Chew EH and Holmgren A: Targeting thioredoxin reductase is a basis for cancer therapy by arsenic trioxide. Proc Natl Acad Sci USA 104: 12288-12293, 2007.

31. States JC, Srivastava S, Chen Y and Barchowsky A: Arsenic and cardiovascular disease. Toxicol Sci 107: 312-323, 2009.

32. Rahman MM, Ng JC and Naidu R: Chronic exposure of arsenic via drinking water and its adverse health impacts on humans. Environ Geochem Health 31 (Suppl 1): S189-S200, 2009.

33. Rahman M, Tondel M, Ahmad SA, Chowdhury IA, Faruquee MH and Axelson O: Hypertension and arsenic exposure in Bangladesh. Hypertension 33: 74-78, 1999.

34. Tseng CH: Cardiovascular disease in arsenic-exposed subjects living in the arseniasis-hyperendemic areas in Taiwan. Atherosclerosis 199: 12-18, 2008.

35. Islam LN, Nabi AH, Rahman MM and Zahid MS: Association of respiratory complications and elevated serum immunoglobulins with drinking water arsenic toxicity in human. J Environ Sci Health A Tox Hazard Subst Environ Eng 42: 1807-1814, 2007.

36. Park WH, Han BR, Park HK and Kim SZ: Arsenic trioxide induces growth inhibition and death in human pulmonary artery smooth muscle cells accompanied by mitochondrial $\mathrm{O} 2{ }^{\bullet}$ increase and GSH depletion. Environ Toxicol, 2018.

37. You BR and Park WH: Suberoylanilide hydroxamic acid induces thioredoxin1-mediated apoptosis in lung cancer cells via up-regulation of miR-129-5p. Mol Carcinog 56: 2566-2577, 2017.

38. You BR and Park WH: Down-regulation of thioredoxin1 is involved in death of Calu-6 lung cancer cells treated with suberoyl bishydroxamic acid. J Cell Biochem 117: 1250-1261, 2016.

39. You BR, Kim SH and Park WH: Reactive oxygen species, glutathione, and thioredoxin influence suberoyl bishydroxamic acid-induced apoptosis in A549 lung cancer cells. Tumour Biol 36: 3429-3439, 2015.
40. Park WH: Pyrogallol induces the death of human pulmonary fibroblast cells through ROS increase and GSH depletion. Int J Oncol 49: 785-792, 2016.

41. Berridge MV, Herst PM and Tan AS: Tetrazolium dyes as tools in cell biology: New insights into their cellular reduction. Biotechnol Annu Rev 11: 127-152, 2005.

42. Han YH, Kim SH, Kim SZ and Park WH: Caspase inhibitor decreases apoptosis in pyrogallol-treated lung cancer Calu-6 cells via the prevention of GSH depletion. Int $\mathbf{J}$ Oncol 33: 1099-1105, 2008

43. Arnér ES and Holmgren A: The thioredoxin system in cancer. Semin Cancer Biol 16: 420-426, 2006.

44. Fernandes AP, Capitanio A, Selenius M, Brodin O, Rundlöf AK and Björnstedt M: Expression profiles of thioredoxin family proteins in human lung cancer tissue: Correlation with proliferation and differentiation. Histopathology 55: 313-320, 2009.

45. Sugiura $H$ and Ichinose $M$ : Oxidative and nitrative stress in bronchial asthma. Antioxid Redox Signal 10: 785-797, 2008.

46. You BR and Park WH: Suberoylanilide hydroxamic acid-induced HeLa cell death is closely correlated with oxidative stress and thioredoxin 1 levels. Int J Oncol 44: 1745-1755, 2014.

47. Schenk H, Klein M, Erdbrügger W, Dröge $W$ and Schulze-Osthoff K: Distinct effects of thioredoxin and antioxidants on the activation of transcription factors NF-kappa B and AP-1. Proc Natl Acad Sci USA 91: 1672-1676, 1994.

48. Hirota K, Murata M, Sachi Y, Nakamura H, Takeuchi J, Mori K and Yodoi J: Distinct roles of thioredoxin in the cytoplasm and in the nucleus. A two-step mechanism of redox regulation of transcription factor NF-kappaB. J Biol Chem 274: 27891-27897, 1999.

49. Bloomfield KL, Osborne SA, Kennedy DD, Clarke FM and Tonissen KF: Thioredoxin-mediated redox control of the transcription factor $\mathrm{Sp1}$ and regulation of the thioredoxin gene promoter. Gene 319: 107-116, 2003.

50. Daloso DM, Müller K, Obata T, Florian A, Tohge T, Bottcher A, Riondet C, Bariat L, Carrari F, Nunes-Nesi A, et al: Thioredoxin, a master regulator of the tricarboxylic acid cycle in plant mitochondria. Proc Natl Acad Sci USA 112: E1392-E1400, 2015.

51. Jin Y, Zhang X, Shu L, Chen L, Sun L, Qian H, Liu W and Fu Z: Oxidative stress response and gene expression with atrazine exposure in adult female zebrafish (Danio rerio). Chemosphere 78: 846-852, 2010.

52. Sarkar S, Mukherjee S, Chattopadhyay A and Bhattacharya S: Differential modulation of cellular antioxidant status in zebrafish liver and kidney exposed to low dose arsenic trioxide. Ecotoxicol Environ Saf 135: 173-182, 2017.

53. Kim HR, Kim EJ, Yang SH, Jeong ET, Park C, Kim SJ, Youn MJ, So HS and Park R: Combination treatment with arsenic trioxide and sulindac augments their apoptotic potential in lung cancer cells through activation of caspase cascade and mitochondrial dysfunction. Int J Oncol 28: 1401-1408, 2006.

54. Sarkar S, Mukherjee S, Chattopadhyay A and Bhattacharya S: Low dose of arsenic trioxide triggers oxidative stress in zebrafish brain: Expression of antioxidant genes. Ecotoxicol Environ Saf 107: 1-8, 2014

55. Dai J, Weinberg RS, Waxman S and Jing Y: Malignant cells can be sensitized to undergo growth inhibition and apoptosis by arsenic trioxide through modulation of the glutathione redox system. Blood 93: 268-277, 1999.

56. Han YH, Kim SZ, Kim SH and Park WH: Induction of apoptosis in arsenic trioxide-treated lung cancer A549 cells by buthionine sulfoximine. Mol Cells 26: 158-164, 2008

57. Han YH, Kim SH, Kim SZ and Park WH: Apoptosis in arsenic trioxide-treated Calu- 6 lung cells is correlated with the depletion of GSH levels rather than the changes of ROS levels. J Cell Biochem 104: 862-878, 2008. 